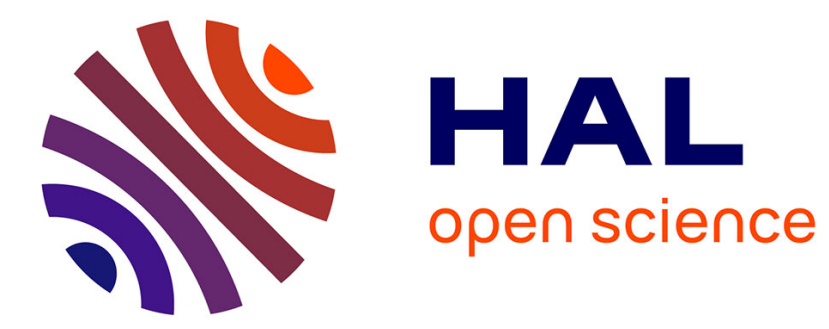

\title{
A Parametric Active Polygon for Leaf Segmentation and Shape Estimation
}

Guillaume Cerutti, Laure Tougne, Antoine Vacavant, Didier Coquin

\section{To cite this version:}

Guillaume Cerutti, Laure Tougne, Antoine Vacavant, Didier Coquin. A Parametric Active Polygon for Leaf Segmentation and Shape Estimation. 7th International Symposium on Visual Computing, Sep 2011, Las Vegas, United States. pp.202-213. hal-00622269

\section{HAL Id: hal-00622269 https://hal.science/hal-00622269}

Submitted on 12 Sep 2013

HAL is a multi-disciplinary open access archive for the deposit and dissemination of scientific research documents, whether they are published or not. The documents may come from teaching and research institutions in France or abroad, or from public or private research centers.
L'archive ouverte pluridisciplinaire $\mathbf{H A L}$, est destinée au dépôt et à la diffusion de documents scientifiques de niveau recherche, publiés ou non, émanant des établissements d'enseignement et de recherche français ou étrangers, des laboratoires publics ou privés. 


\title{
A Parametric Active Polygon for Leaf Segmentation and Shape Estimation ${ }^{\star}$
}

\author{
Guillaume Cerutti $^{1,2}$, Laure Tougne ${ }^{1,2}$, Antoine Vacavant $^{3}$, Didier Coquin ${ }^{4}$ \\ 1 Université de Lyon, CNRS \\ 2 Université Lyon 2, LIRIS, UMR5205, F-69676, France \\ 3 Université d'Auvergne, ISIT, F-63000, Clermont-Ferrand \\ ${ }^{4}$ LISTIC, Domaine Universitaire, F-74944, Annecy le Vieux
}

\begin{abstract}
In this paper we present a system for tree leaf segmentation in natural images that combines a first, unrefined segmentation step, with an estimation of descriptors depicting the general shape of a simple leaf. It is based on a light polygonal model, built to represent most of the leaf shapes, that will be deformed to fit the leaf in the image. Avoiding some classic obstacles of active contour models, this approach gives promising results, even on complex natural photographs, and constitutes a solid basis for a leaf recognition process.
\end{abstract}

\section{Introduction}

Over the past years of progress and urbanization, the world of plants has lost the part it formerly had in our everyday life, and the names and uses of the many trees, flowers and herbs that surround us now constitute a knowledge accessible only to botanists. But nowadays, with the rising awareness that plant resources and diversity ought to be treasured, the will to regain some touch with nature never felt so present. And what better tool to achieve this than the ubiquitous mobile technology, that has the opportunity of placing a flora book in everyones pocket? Botanists traditionally rely on the aspect and composition of fruits, flowers and leaves to identify species. But in the context of a widespread nonspecialist-oriented application, the predominant use of leaves, which are possible to find almost all year long, simple to photograph, and easier to analyze, is the most sensible and widely used approach in image processing. Considering the shape of a leaf is then the obvious choice to try to recognize the species. Starting by tree leaves which are the easiest to spot, our goal is to build a system to retrieve relevant geometric criteria to classify a leaf, in a photograph taken by a smartphone camera.

In this paper we present a method to achieve a first part of this process, estimating the general shape of a leaf that will guide the segmentation process. In Section 2 we present the related publications. Section 3 expounds the model used to represent the general shape of a leaf, and Section 4 the so-called parametric

\footnotetext{
* This work has been supported by the French National Agency for Research with the reference ANR-10-CORD-005 (REVES project).
} 
active polygon segmentation algorithm we use. In Section 5, we show how this system could be used for leaf shape classification. Section 6 relates the results of experiments.

\section{Related work}

Plant recognition has recently been a subject of interest for various works. Few of them though consider acquiring images of leaves or flowers in a complex, natural environment, thus eluding most of the hard task of segmentation.

\subsection{Plant recognition and segmentation}

Nilsback and Zisserman [1] adressed the problem of segmenting flowers in natural scenes, by using a geometric model, and classifying them over a large number of classes. Saitoh and Kaneko 2 focus also on flowers, but in images with hard constraints on out-of-focus background. Such approaches are convenient for flowers, but lose much of their efficiency with leaves.

Many works on plant leaf recognition tend to avoid the problem by using a plain sheet of paper to make the segmentation easy as pie. Their recognition systems are then based on either statistical or geometric features : CentroidContour Distance (CCD) curve [3], moments [43], histogram of gradients, or SIFT points [1. Some more advanced statistical descriptors, such as the Inner Distance Shape Context [5], or the Curvature Scale Space representation [6] that allows taking self-intersections into account, have also been applied to the context of leaf classification, while developed in a general purpose.

As a matter of fact, isolating green leaves in an overall not less green environment seems like a much tougher issue, and only some authors have designed algorithms to overcome the difficulties posed by a natural background. Teng, Kuo and Chen [7] used 3D points reconstruction from several different images to perform a 2D/3D joint segmentation using 3D distances and color similarity. Wang [4 performed an automatic marker-based watershed segmentation, after a first thresholding-erosion process. All these approaches are complex methods that seem hardly reachable for a mobile application. In the case of weed leaves, highly constrained deformable templates have been used 8 to segment one single species, providing good results even with occlusions and overlaps.

\subsection{Active contour models}

The concept of active contours, or snakes, have been introduced by Kass, Witkin and Terzopulos 9 as a way to solve problems of edge detection. They are splines that adjust to the contours in the image by minimizing an energy functional. This energy is classically composed of two terms, an internal energy term considering the regularity and smoothness of the desired contour, and an external or image 
energy accounting for its adequation with the actual features in the image, based on the intensity gradient.

To detect objects that are not well defined by gradient, Chan and Vese 10. for instance based the evolution of their active contour on the color consistency of the regions, using a level set formulation. Another region-based approach, relying this time on texture information, was proposed by Unal, Yezzi and Krim 11. who also introduced a polygonal representation of the contour.

But to include some knowledge about complex objects, a deformable template 12 can be used, with the asset of lightening the representation and storage space of the contour by the use of parameters. Felzenszwalb [13] represents shapes by deformable triangulated polygons to detect precisely described objects, including maple leaves, and Cremers [14] includes shape priors into level set active contours to segment a known object, but both approaches lack the flexibility needed to include knowledge about the shape of any possible leaf.
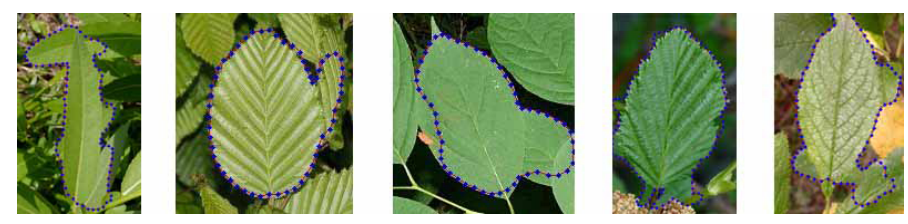

Fig. 1. Segmentation issues with classic region-based active contour model

In our case, the similarity between the background and the object of interest, and the difficulty to avoid adjacent and overlapping leaves constitute a prohibitive obstacle to the use of unconstrained active contours (Figure 1). The idea of using a template to represent the leaves is complicated by the fact that there is much more variety in shapes than for eyes or mouths. The only solution to overcome the aforementioned problems is however to take advantage of the prior knowledge we may have on leaf shapes to design a very flexible time-efficient model to represent leaves.

\section{A polygonal model for simple tree leaves}

Despite the large variety in leaf shapes, even when considering only the trees, it is necessary to come up with some kind of template that can rather accurately be fitted to basically any kind of leaf. We considered in a first time only trees with simple, non-palmate leaves, which represent about $80 \%$ of French broad-leaved tree species [15] and roughly the same proportion in all European species.

\subsection{Describing botanic leaf shapes}

Botanists have a specialized vocabulary to describe leaf shapes, among which around 15 terms are used to characterize the global shape of a leaf. Examples of 
such shapes are diplayed in Figure 2. The interesting point is that these terms are also the ones used in flora books to describe the lobes of palmate leaves and the leaflets of compounded leaves, making them the base element to describe all the leaf shapes.

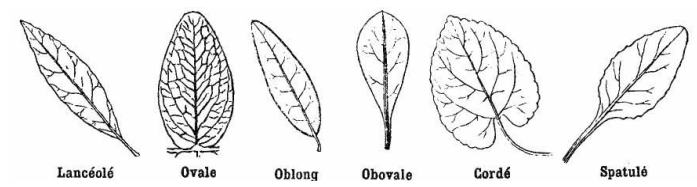

Fig. 2. Examples of leaf shapes used by botanists : Lanceolate, Ovate, Oblong, Obovate, Cordate and Spatulate. Images taken from [15].

Knowing this general shape is a key component of the process of identifying a leaf, though non sufficient. However, given the high variability within a single species, floras often reference a species with two or three leaf shapes, making this notion overall blurry. This impression is reinforced by the difficulties in seeing where exactly a denomination ends and the other one begins.

It is yet possible to derive a basic model to sketch all the shapes used in botany. The idea is then to use a light polygonal model, suitable for a time and space effective mobile implementation. It is only assumed to have a vertical axis of symmetry joining the base and the tip, and from this, by playing on a set of parameters, one should be able to describe the whole set of shape types.

This kind of representation has two main advantages. First the parameters that define the construction of the model can later be directly used as descriptors for the leaf, and using such representation in segmenting leaves is a way to create a cooperation between the two classically unconnected processes of segmentation and feature extraction. Additonally, working on numeric values allows us to slightly reduce the uncertainty produced by the hard classification into not-welldefined botanical terms of natural objects, which intrinsically bear a part of variability.

\subsection{The parametric polygonal leaf template}

The chosen model relies on two points, base $B$ and tip $T$, that define the main axis of the leaf. From this axis, we construct the 10 points defining the polygon, using 4 numeric integer parameters :

$-\alpha_{B}$, the opening angle at the base

$-\alpha_{T}$, the opening angle at the tip

$-w$, the relative maximal width

$-p$, the relative position where this width is reached

The model is then built as follows. In the next steps, we will call $\boldsymbol{a}$ the direction vector of the line segment $[B T], \boldsymbol{n}$ its normal vector, and $h$ its length, so that $\overrightarrow{B T}=$ h.a. The construction of the model is illustrated in Figure 3 

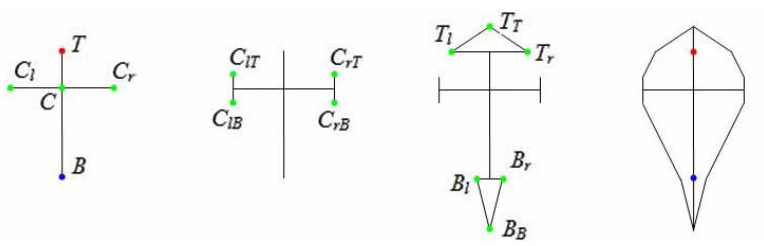

Fig. 3. Building the leaf model in 4 steps

1. The center point $C$ is defined as $\boldsymbol{B} \boldsymbol{C}=p \cdot \boldsymbol{B} \boldsymbol{T}$ and perpendicularly to the main axis is drawn the segment of maximal width $\left[C_{l} C_{r}\right]$, centered on $C$. In other terms, we have $\overrightarrow{C C_{l}}=\frac{w}{2} h . \boldsymbol{n}$ and $\overrightarrow{C C_{r}}=-\frac{w}{2} h . \boldsymbol{n}$.

2. At the points $C_{l}$ and $C_{r}$ we trace two tangents, whose length depend on the height and the parameter p, defining 4 new points $C_{l B}, C_{l T}, C_{r B}$ and $C_{r T}$. For instance, $\overrightarrow{C_{l} C_{l T}}=(1-2|p-0.5|) \frac{h}{5} \cdot \boldsymbol{a}$ and $\overrightarrow{C_{l} C_{l B}}=-(1-2|p-0.5|) \frac{h}{5} \cdot \boldsymbol{a}$, and the same is done for the right tangent.

3. At the tip and at the base, we build two isosceles triangles, with a top angle respectively of $\alpha_{T}$ and $\alpha_{B}$ and with equal sides of fixed length relatively to the height. This creates 6 additional points $T_{l}, T_{r}$ and $T_{T}$ for the tip and $B_{l}, B_{r}$ and $B_{B}$ for the base. The points at the tip are defined by $\overrightarrow{T T}_{l}=$ $\frac{h}{5} \sin \frac{\alpha_{T}}{2} \cdot \boldsymbol{n}, \overrightarrow{T T_{r}}=-\frac{h}{5} \sin \frac{\alpha_{T}}{2} \cdot \boldsymbol{n}$ and $\overrightarrow{T T_{T}}=\frac{h}{5} \cos \frac{\alpha_{T}}{2} \cdot \boldsymbol{a}$ and the points at the base are defined identically.

4. Finally, the polygon is obtained by linking the 10 points $B_{B}, B_{l}, C_{l_{B}}, C_{l_{T}}$, $T_{l}, T_{T}, T_{r}, C_{r_{T}}, C_{r_{B}}$ and $B_{r}$.

This model proves to cover, with a very restraint set of parameters, the major part of the shapes used by botanists to describe leaves. It is then possible to adjust the parameters to recreate models that visibly correspond to most of the archetypal leaf shapes, as depicts Figure 4. Other parameters could have been added to account for deformations that occur in real images, but it would have compromised the lightness of the model.

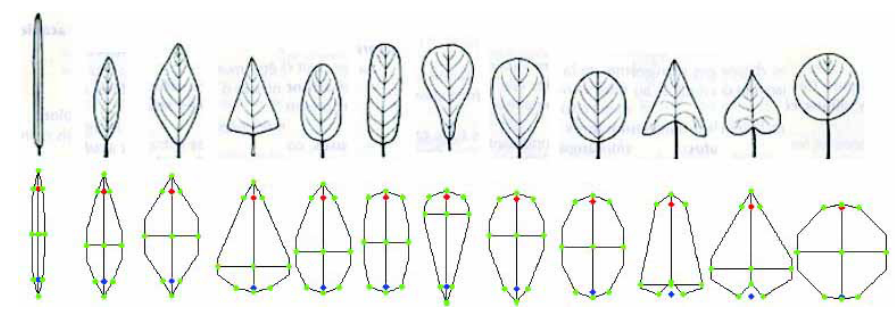

Fig. 4. Main leaf shapes and their corresponding hand-tuned models 


\section{Parametric Active Polygon}

As mentioned earlier, using a deformable template approach based on this model has the advantage of encapsulating some prior knowledge on the shape of the object in the very definition of the model. It is also a great benefit in terms of computation time to have a parametric representation, as there will be only a few elementary deformations, making the exploration of the possible variations easier.

\subsection{Elementary deformations}

The values that can be changed to deform the model are very few since the considered polygon is entirely defined by the indication of the 2 points defining the main axis and the 4 integer parameters. Consequently, a given model will have only 16 neighbours in the model space, corresponding to a variation in one direction or the other of a parameter ( 8 deformations) and the move of a point to one of its neighbours in the image in the sense of 4-connectivity ( 8 deformations).

This results in a very limited set of elementary variations to be considered when deforming the model, compared with a classic active contour that can be moved pointwise locally.

\subsection{Representing the color}

The polygonal model being an approximation of a shape, we can not expect it to fit edges in the image. That is why we use a region-based approach, using color information inside the region rather than gradient information on its contour. But formulating an a priori model for color, that would be accurate whatever the leaf, season and lighting is likely an impossible task.

It is therefore an absolute necessity to learn the particular model for the leaf we want to segment from the image. And this can obviously only be achieved with some constraints on the position of the leaf, that will allow us to know where to evaluate a color model. In the following, we assume that the leaf is roughly centered and vertically-oriented, so that an initialization of our template in the middle of the image contains almost only leaf pixels. Typically, a leaf in the image taken on purpose by a user will be large enough to ensure the correctness of the initialization.

We considered then that the color of a leaf can be modelled by a 2-component GMM estimated in the initial region, accounting for shaded and lighted or shiny areas, and defined by the parameters $\left(\mu_{1}, \sigma_{1}^{2}, \alpha_{1}\right)$ and $\left(\mu_{2}, \sigma_{2}^{2}, \alpha_{2}\right)$, respectively the mean, variance and weight of each gaussian distribution. Then the distance of a pixel $x$ to the color model, in a 3-dimensional colorspace, is defined by a Mahalanobis 1-norm distance, considering the variance matrices are diagonal, written as following : $d\left(x, \mu_{1,2}, \sigma_{1}, \mu_{2}, \sigma_{2}\right)=\min _{g=1,2} \sum_{i=1}^{3} \frac{\left|x_{i}-\mu_{g, i}\right|}{\sigma_{g, i}}$

Based on this formulation, we can compute for every pixel in the image its distance to the color model, resulting in a distance map, where leaf pixels should 
appear in black and background pixels in gray-white. Based on the aspect of this map for different leaves and different color spaces, we chose to work in the Lab colorspace, for which leaves were standing out best in distance maps.

\subsection{Energy functional}

The internal energy term that classically appears in the energy formulation can be considered as implicit here, as it is included in the construction rules of our model, giving it already some kind of rigidity. The remaining term is the external energy, representing the image forces, and it will be based on the distance map discussed above, as we ultimately want the final polygon to contain only pixels with as small distance to the leaf color model as possible. The energy the model strives to minimize throughout its evolution is then expressed as :

$$
E(M)=\sum_{x \in M}\left(d\left(x, \mu_{1}, \sigma_{1}, \mu_{2}, \sigma_{2}\right)-d_{\max }\right)
$$

The value $d_{\max }$ actually represents a balloon force, that will push the model to grow as much as possible. It can also be seen as a threshold, the distance to the color model for which a pixel will be costly to add into the polygon. The expected outcome is to produce the biggest region with as little color-distant pixels as possible.

At each step, the algorithm will then select, among the 16 possible elementary variations, the one that leads to the most important decrease of the energy, until no deformation can bring it any lower. As such, this method has the major drawback of getting stuck in local energy minima, that most certainly do not correspond to the searched leaf. To get around this problem, we use a heuristic close to simulated annealing.

\subsection{Evolution constraints}

Even if the polygonal template models quite accurately most of the leaf shapes, it has the unwanted counterpart of being slightly too flexible, and thus able to model shapes that are not likely to ever be reached by leaves. This has negative consequences when the model is let to evolve freely, at will undergo any possible deformation to minimize its energy, often resulting in unnatural, yet surprising, optimal shapes.

It is necessary then to constraint the evolution of the template to make sure it keeps an acceptable leaf shape. To achieve this, the model is forced to have its parameters within an authorized range. This range have been learned by manually adjusting the model to leaves from a databas $£^{5}$ A template can then be told as leaf or non-leaf by estimating its distance to the empiric leafmodel in the parameter space. Only the variations that give a leaf template are then examinated in the evolution process, ensuring the final polygon will show a satisfactory shape. The Figure 5 shows sample results of the whole process.

\footnotetext{
${ }^{5}$ LEAF - Tree Leaf Database, Inst. of Information Theory and Automation ASCR, Prague, Czech Republic, http://zoi.utia.cas.cz/tree_leaves
} 

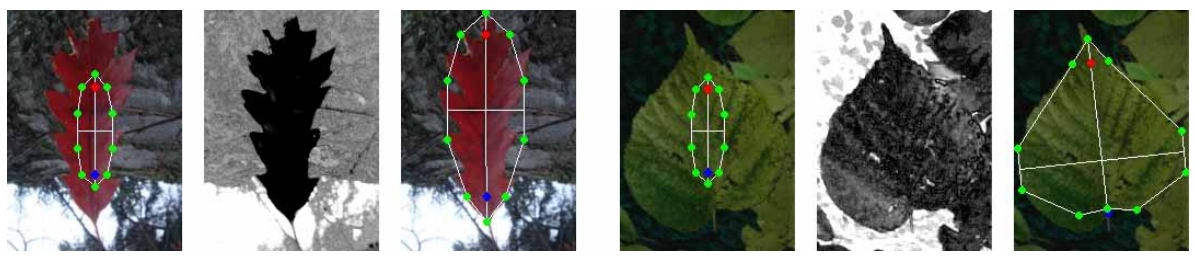

Fig. 5. Initialization, distance map and resulting polygon on two natural images

\section{Shape Classification}

To measure with more accuracy the performance of our approach, we use the parameters returned by the algorithm as descriptors to estimate the global leaf shape. It is important to keep in mind though that the final goal is to recognize a plant species, and that the numeric parameter values convey more information than a rigid classification into rather unclear terms. These constitute actually global descriptors that will later be combined with local geometric features and other heteregenous data such as date or GPS coordinates to recognize species. This shape classification step will consequently not even be a middle step in our process, but it appeared as the best way to evaluate the behaviour of our algorithm.

\section{$5.1 \quad$ Learning leaf shapes}

The first step is to learn the different classes of leaf shapes and the parameter values they correspond to. As discussed earlier, the problem is that the botanic classification is a very subjective and uncertain work, the boundaries between classes being vaguely defined, and a tree species impossible to associate with a single class. It is then judicious to let an almost unsupervised learning algorithm determine the best classification, based of the set of parameters manually extracted from the leaf database ${ }^{5}$. This is simply performed by a $k$-Means clustering algorithm that classifies points in the parameters space, and returns $k$ classes, each one represented by its centroid and its variance for each parameter. The value of $k$ that produced the best, visibly identifiable, models was $k=10$. The clustering was however guided by initializing the centroids on hand-designed leaf shape archetypes, but was then left free to evolve. This way, we obtain ten canonical models, displayed in Figure 6 that can later be labeled by the name of the botanic class(es) they match best.

\section{$5.2 k$-Means classification}

The model resulting from the parametric active polygon algorithm can then be compared to each of the class centroids, by computing a 1-norm distance in the parameters space. As illustrated in Figure 7 the model is labeled with the closest 

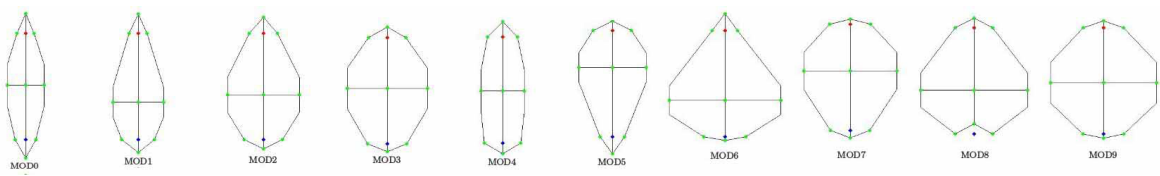

Fig. 6. The 10 models representing the classes of leaf shapes used for classification
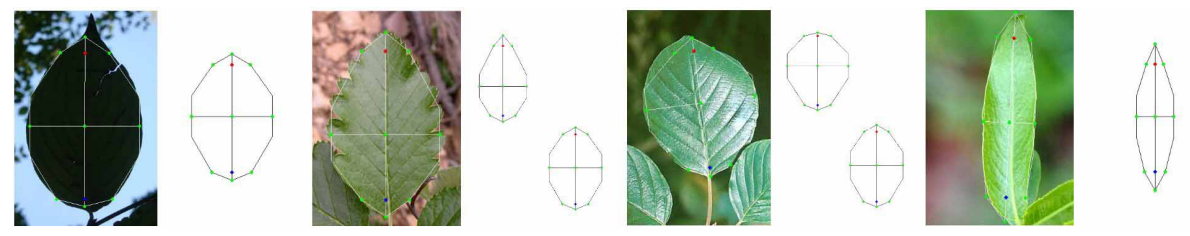

Fig. 7. Obtained models and closest leaf shape centroids

class $k^{*}$, or with the two closest ones if $\min _{k \neq k^{*}}\left\|M-M_{k}\right\|_{1}<\beta \cdot \min _{k}\left\|M-M_{k}\right\|_{1}$ $(\beta>1)$.

It is actually frequent that the shape of a leaf can not be assigned to one class only, even by an experimented botanist. Returning often 2 canonical shapes as a classification result is therefore not strictly speaking approximative, but rather complying with a natural reality.

\section{Experiments \& Results}

\subsection{Experimental procedure}

Tests were performed on two databases : a white-background leaf databas $\AA^{6}$ and a set of natural scene leaf images, centered and rotated to fit the desired conditions. Unfortunately, we have not yet collected images from smartphone cameras, taken by users from a prototypal interface, which would correspond best to the images the algorithm was designed to work on.

Each image is then set to roughly the same size and the model launched on it, to estimate the shape of the leaf. A ground-truth class labeling was first performed by a group of about 15 specialists and non-specialists provided with the images and the 10 class models. Each image was then associated with one or two ordered classes depending on the votes of the labelers, and the classification in turn responds with one or two classes.

\subsection{Performance measure}

Measuring the accuracy of such answers, consisting most of the time in an ordered pair of classes to be compared with an other ordered pair, is not a common

6 Database collected by P.-Y. Landouer on his website http://www.lesarbres.fr 
problem, and there does not seem to be a standard procedure to do so. We tried however to build simply a confusion matrix estimating how fitting the answer is.

If the first actual class is present among the two predicted ones, 2 points are placed on the corresponding diagonal cell. If the second actual class is present, 1 point is placed on the corresponding diagonal cell, and if the first class was not recognized, 1 point is placed on the cell from the line of the first class, in the column of the other recognized class. And finally, if none on the classes are present, 1 point is placed on each of the cells from the lines corresponding to the actual classes, in the columns corresponding to the predicted classes. The points are then normalized to have their total equal to 1.

Table 1. Confusion matrix for 99 white background images

\begin{tabular}{|l|l|l|l|l|l|l|l|l|l|l|}
\hline & M0 & M1 & M2 & M3 & M4 & M5 & M6 & M7 & M8 & M9 \\
\hline M0 & 100 & 0 & 0 & 0 & 0 & 0 & 0 & 0 & 0 & 0 \\
\hline M1 & 6 & 93 & 0 & 0 & 0 & 0 & 0 & 0 & 0 & 0 \\
\hline M2 & 0 & 0 & 92 & 5 & 0 & 0 & 0 & 0 & 0 & 2 \\
\hline M3 & 0 & 0 & 1 & 94 & 0 & 0 & 0 & 2 & 0 & 2 \\
\hline M4 & 0 & 0 & 0 & 0 & 100 & 0 & 0 & 0 & 0 & 0 \\
\hline M5 & 0 & 0 & 3 & 0 & 0 & 96 & 0 & 0 & 0 & 0 \\
\hline M6 & 0 & 0 & 0 & 0 & 0 & 0 & 93 & 0 & 3 & 3 \\
\hline M7 & 0 & 0 & 0 & 0 & 0 & 0 & 0 & 100 & 0 & 0 \\
\hline M8 & 0 & 0 & 3 & 0 & 0 & 0 & 3 & 0 & 92 & 0 \\
\hline M9 & 0 & 0 & 0 & 0 & 0 & 0 & 0 & 4 & 0 & 95 \\
\hline
\end{tabular}

Table 2. Confusion matrix for 113 natural scene images

\begin{tabular}{|l|l|l|l|l|l|l|l|l|l|l|}
\hline & M0 & M1 & M2 & M3 & M4 & M5 & M6 & M7 & M8 & M9 \\
\hline M0 & 87 & 5 & 2 & 0 & 5 & 0 & 0 & 0 & 0 & 0 \\
\hline M1 & 0 & 81 & 0 & 5 & 8 & 2 & 0 & 2 & 0 & 2 \\
\hline M2 & 0 & 0 & 79 & 8 & 0 & 4 & 0 & 2 & 0 & 6 \\
\hline M3 & 1 & 0 & 6 & 82 & 2 & 2 & 2 & 0 & 3 & 3 \\
\hline M4 & 0 & 0 & 13 & 8 & 79 & 0 & 0 & 0 & 0 & 0 \\
\hline M5 & 0 & 0 & 1 & 5 & 0 & 88 & 0 & 5 & 0 & 0 \\
\hline M6 & 1 & 3 & 8 & 8 & 0 & 0 & 76 & 0 & 2 & 2 \\
\hline M7 & 10 & 10 & 0 & 0 & 0 & 0 & 0 & 80 & 0 & 0 \\
\hline M8 & 3 & 4 & 12 & 3 & 0 & 0 & 3 & 0 & 75 & 3 \\
\hline M9 & 2 & 2 & 0 & 5 & 0 & 0 & 13 & 5 & 0 & 73 \\
\hline
\end{tabular}

In the case of white background images (Table 1) the model shows a very good classification rate, and recognizes one of the classes in almost every case. Some classes already seem hard to discriminate like the M2 (Ovate) and the M3 (Elliptic) as it is a problem even for humans when to say ovate or elliptic. Another tendency is to label Cordate (M8) some Triangular leaves (M6). This may come from the very definition of the polygonal model that has difficulties fitting exactly round shapes, and sometimes, a heart-shaped base allows it to get closer to the actual border of a triangular leaf. This issue results in a wrong classification but remains close to the truth in terms in terms of segmentation.

For natural images (Table 2 ) the overall performance is obviously lower, but remains acceptable. The main problem is the variability of the color inside the leaf, factor that does not exist on white background images thanks to uniform controlled lighting. This often leads the model to segment accurately part of the leaf but not to enter in the areas that are too far from the color model, resulting in a bad classication though the segmentation result would still be exploitable. The same tendencies appear, notably the difficulty to discriminate between Elliptic and Ovate, Ovate and Triangular, or Triangular and Orbicular (M9).

\subsection{Comparison with standard active contours}

The main advantage our model proves to have over a regular snake algorithm based on the same energy term is that it is not sensitive to overflowing. The hard constraints contained in the definition and the evolution of the model impose that it remains in an acceptable shape and forbids any attemps of exploring unwanted 
regions. This is a guarantee that the final shape will not have outgrown out of the leaf in some places, and is a substantial condition for the robustness of the rest of the process.

It will also be more robust with respect to holes or spots inside the leaf that will not change the shape as much as it would with a contour that could go around them. Such irregularities are smoothed by the fact that we consider a whole region of fixed shape.
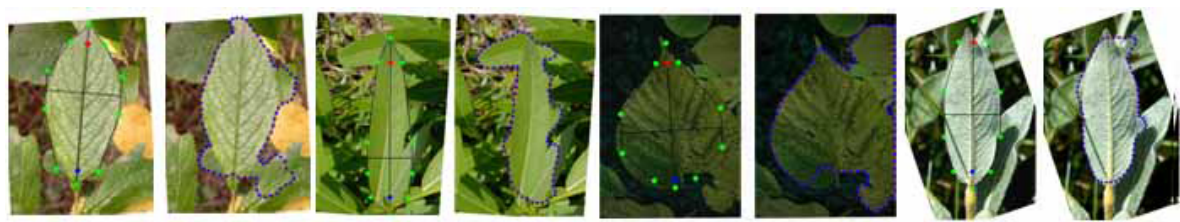

Fig. 8. Comparison between our polygonal model and standard active contour [16]

\section{Conclusions}

We have presented a method to perform a first segmentation of a leaf in a natural scene, based on the optimization of a polygonal leaf model whose parameters constitute also a first set of descriptors with an eye to species identification. The model is flexible and covers most of the simple tree leaf shapes, and is a good base for the segmentation of lobed and compound leaves. It is however rigid enough to avoid classic problems of active contour approaches, that allow for instance overflows to adjacent similar regions, and make use of a color model that is robust to uncontrolled lighting conditions.

Improvements are to be considered. In some cases, relying only on color information does not seem to be the best choice ; the model could benefit from the use of an additional texture model of the leaf. As for the color model, 2 gaussians may not be enough to represent the diversity of the colors in a single leaf. A model with an adaptive number of gaussians is a possible enhancement.

The next step is then to refine the contour of the obtained polygon to fit the actual contour of the leaf in the image. The idea would be to use an active contour this time, constraining it to remain within a certain distance to the polygon. With a more accurate segmentation, we will be able to extract the local geometric features that characterize leaves, and to begin classifying leaf images into species. The model should also be extended to take into account lobed and compound tree leaves, and even non-tree leaves, and be tested over images from smartphone cameras.

Nevertheless, it constitutes as such a good approach to estimate the shape of a leaf in a natural environment, without performing a thorough segmentation, and to associate the corresponding terms used by botanists to describe leaves. 


\section{References}

1. Nilsback, M.E., Zisserman, A.: Delving into the whorl of flower segmentation. In: British Machine Vision Conference. Volume 1. (2007) 570-579

2. Saitoh, T., Kaneko, T.: Automatic recognition of blooming flowers. International Conference on Pattern Recognition 1 (2004) 27-30

3. Wang, Z., Chi, Z., Feng, D., Wang, Q.: Leaf image retrieval with shape features. In: Advances in Visual Information Systems. Volume 1929 of Lecture Notes in Computer Science. (2000) 41-52

4. Wang, X.F., Huang, D.S., Du, J.X., Huan, X., Heutte, L.: Classification of plant leaf images with complicated background. Applied Mathematics and Computation 205 (2008) 916-926

5. Belhumeur, P., Chen, D., Feiner, S., Jacobs, D., Kress, W., Ling, H., Lopez, I., Ramamoorthi, R., Sheorey, S., White, S., Zhang, L.: Searching the world's herbaria: A system for visual identication of plant species. In: European Conference on Computer Vision. (2008)

6. Mokhtarian, F., Abbasi, S.: Matching shapes with self-intersections: Application to leaf classification. IEEE Transactions on Image Processing 13 (2004) 653-661

7. Teng, C.H., Kuo, Y.T., Chen, Y.S.: Leaf segmentation, its 3d position estimation and leaf classification from a few images with very close viewpoints. In: Proceedings of the 6th International Conference on Image Analysis and Recognition. ICIAR '09 (2009) 937-946

8. Manh, A.G., Rabatel, G., Assemat, L., Aldon, M.J.: Weed leaf image segmentation by deformable templates. Journal of agricultural engineering research 80 (2001) $139-146$

9. Kass, M., Witkin, A., Terzopoulos, D.: Snakes: Active contour models. International Journal of Computer Vision 1 (1988) 321-331

10. Chan, T., Vese, L.: Active contours without edges. IEEE Transactions on Image Processing 10 (2001) 266-277

11. Unal, G., Yezzi, A., Krim, H.: Information-theoretic active polygons for unsupervised texture segmentation. International Journal of Computer Vision 62 (2005) 199-220

12. Yuille, A., Hallinan, P., Cohen, D.: Feature extraction from faces using deformable templates. International Journal of Computer Vision 8 (1992) 99-111

13. Felzenszwalb, P.: Representation and detection of deformable shapes. PAMI 27 (2004) 208-220

14. Cremers, D., Tischhuser, F., Weickert, J., Schnrr, C.: Diffusion snakes: introducing statistical shape knowledge into the mumford-shah functional. Journal Of Computer Vision 50 (2002) 295-313

15. Coste, H.: Flore descriptive et illustrée de la France de la Corse et des contrées limitrophes. (1906)

16. Mille, J.: Narrow band region-based active contours and surfaces for $2 \mathrm{~d}$ and $3 \mathrm{~d}$ segmentation. Computer Vision and Image Understanding 113 (2009) 946-965 